\title{
New technique: removal of embedded esophageal partially covered stent by endoscopic sub-stent space dissection
}

\author{
Iyad Khamaysia,c, Sobhi Abadib, Eisa Hajjc, Kamel Yassinc \\ Israel Institute of Technology; Rambam Medical Center, Rambam Health Care Campus, Haifa, Israel
}

\section{Abstract}

\begin{abstract}
Both fully (FCSEMS) and partially (PCSEMS) covered self-expandable metal stents are used for the endoscopic management of leaks, perforations, and fistulas. PCSEMS have lower migration rates as their uncovered flanges allow for anchorage into the surrounding tissue, but this consequently makes them difficult to be removed, with reported cases of esophageal perforation during the removal of the embedded PCSEMS. Stent-in-stent technique is mostly used, where a FCSEMS is placed co-axially into an existing PCSEMS causing pressure necrosis and facilitating subsequent removal of both stents. This technique, although effective, is expensive, requiring the use of a second stent. In this report, we describe a series of 3 patients with embedded PCSEMS removed by a new endoscopic technique, i.e., endoscopic sub-stent space dissection, without using a second stent.
\end{abstract}

Keywords Emdedded partially-covered esophageal stent, sub-stent space dissection, removal

\section{Introduction}

Gastrointestinal (GI) leaks and fistulas are serious, potentially life-threatening surgical complications [1]. Prevalence of GI leaks has increased in recent years most probably due to an increased complexity of GI surgery. Postoperative leaks after oncological surgery have been reported in $8-26 \%$ of the cases after distal esophagectomy and in 3-12\% of the cases after total gastrectomy. Leaks represent a major concern even in bariatric surgery with a prevalence of 1-2\% after sleeve gastrectomy and from 2-8\% after Roux-en-Y gastric bypass [2,3].

Surgical revision had been the mainstay treatment of GI leaks. However, operative interventions may be complex and dangerous [4]. Therefore, an increasing interest in endoscopic management was developed. Recent advances in interventional

aThe Ruth and Bruce Rappaport Faculty of Medicine, Technion, Israel Institute of Technology (Iyad Khamaysi); ' Department of Radiology, Rambam Medical Center (Sobhi Abadi); ' Department of Gastroenterology, Rambam Health Care Campus (Iyad Khamaysi, Eisa Hajj, Kamel Yassin), Haifa, Israel

\section{Conflict of Interest: None}

Correspondence to: Iyad Khamaysi, MD, Director, Invasive Endoscopy Unit, Department of Gastroenterology and Hepatotology, Rambam Health Care Campus, Clinical Assistant Professor, Rappaport Faculty of Medicine, Technion-Israel Institute of Technology, POB 9602, Haifa 31096, Israel, e-mail: iyad.khamaysi@gmail.com

Received 14 September 2020; accepted 22 October 2020; published online 16 January 2021

DOI: https://doi.org/10.20524/aog.2021.0578 endoscopy allowed a paradigm shift in the management leaks from surgery to minimally invasive endoscopic approaches. Therapeutic endoscopy proved to be an effective and less invasive alternative to primary surgery. Available endoscopic treatments include: through-the-scope or over-the-scope clip, stent deployment, endoscopic internal drainage, suturing systems, vacuum assisted therapy, and sealants [5].

Stent insertion has emerged as one of the most effective and safe treatment options for the management of upper GI leaks and fistulas. The rationale of stent deployment is to seal the defect and divert luminal content thus allowing mucosal wall healing. Further advantages include the possibility of early oral intake and reduced risk of stricture formation [6]. Different types of stent may be used, namely: self-expandable plastic and metal stents both fully and partially covered.

Stent migration is a major drawback, since it is responsible for up to one third of cases needing re-intervention, thus increasing costs and adverse events. Fully covered selfexpandable metal stents (FCSEMS) are limited by a relatively high migration rate (33-89\%). Partially covered selfexpandable metal stents (PCSEMS) have been used to allow tissue ingrowth at the edges of the stent, thus decreasing the possibility of migration by its anchorage to the lumen [7].

PCSEMS are mainly designed for malignant esophageal strictures, but are used off-label to close post-surgical leaks due to their lower migration rate than FCSEMS, and their better adherence to the esophageal wall. However, their removal is difficult due to tissue ingrowth through the uncovered part. Nonetheless serious adverse events including segmental esophageal rupture have been described after stent removal [7].

Different methods to remove embedded PCSEMS have been described, the most common being the stent-in-stent 
technique in which a second FCSEMS is deployed inside the embedded one to induce pressure necrosis of hyperplastic tissue ingrowth thus allowing stent removal. Herein we describe a new endoscopic procedure for removal of an embedded PCSEMS without using a second stent.

\section{Case reports}

Three patients with iatrogenic GI wall defect were sent for endoscopic management.

\section{Patient 1}

An 81-year-old female suffering from progressive dysphagia due to benign peptic mid-esophageal stricture underwent an endoscopic balloon dilatation. A 15 -mm wide esophageal perforation was observed. Computed tomography scan of the chest showed a massive pneumomediastinum. Due to her comorbidities, conservative, supportive management was chosen. A PCSEMS (Ultraflex, Boston Scientific Inc., Massachusetts, USA) was then inserted.

\section{Patient 2}

A 55-year-old male with gastric adenocarcinoma underwent a total gastrectomy with esophagoenterostomy. Anastomotic leak was diagnosed 1 week later. A PCSEMS (Wilson-Cook Medical Inc., Winston-Salem, NC, USA) was inserted.

\section{Patient 3}

A 76-year-old male with large mid-esophageal leiomyoma underwent surgical resection of the leiomyoma. During the third post-operative day, a leak with esophago-pleural fistula was diagnosed. Upper endoscopy revealed a $10-\mathrm{mm}$ wide midesophageal perforation. A PCSEMS (Wilson-Cook Medical Inc., Winston-Salem, NC, USA) was inserted.

About 4-6 weeks after the insertion of the stents, an endoscopic procedure to remove the PCSEMSs was performed. In all cases, both uncovered flanges of the stents were

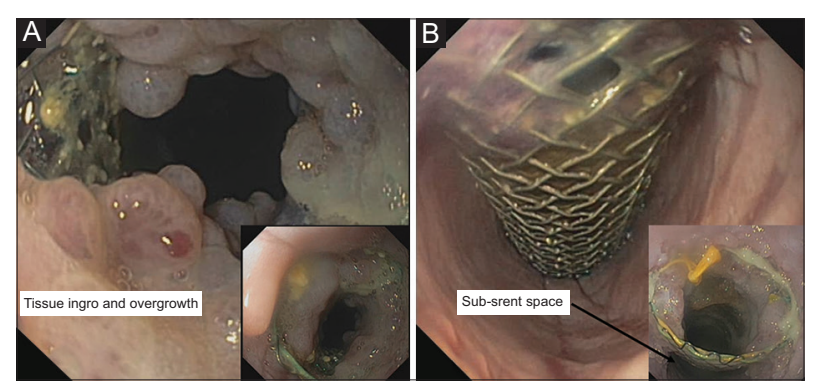

Figure 1 (A) Endoscopic view of tissue ingrowth and overgrowth of the proximal stent flange. (B) Endoscopic view of the sub-stent space completely embedded by extensive, ingrowth and overgrowth of hyperplastic tissue (Fig. 1A). The lasso on both flanges could not be found (also embedded together with the flanges), so it was impossible to remove the stent by pulling out the lasso. Stent-in-stent technique was not performed due to temporary unavailability of FCSEMS.

A trial by the new technique to extract the stents was initiated. The procedures were performed under deep sedation (propofol) in the endoscopy unit. The proximal flange was detached from the esophageal wall using foreign body forceps to make a small opening between the flange of the stent and the esophageal wall. Then, the tip of the gastroscope was carefully advanced in the sub-stent space (the space between the esophageal wall and the stent). Once in the sub-stent space (Fig. 1B, 2A,B), the scope was manipulated in a manner as to dissect the tissue ingrowth around the flange. Multiple gentle circumferential dissection maneuvers were applied to slowly separate and detach the flange from the esophageal wall. Once the proximal flange was separated, the gastroscope was advanced within the sub-stent space down to the distal flange (Fig. 2C). After completing the dissection and separating the distal flange, the proximal or the distal stent lasso was grasped and pulled out (Fig. 2D). Finally, the stent was uneventfully extracted (Fig. 3). No serious bleeding or deep mural injury was encountered apart from only a minor oozing, for which there was no need for hemostatic procedures.

In all 3 cases, the stent was successfully removed (and in the first case, the defect in the GI wall was sealed).

\section{Discussion}

Both FCSEMS and PCSEMS are used for the endoscopic management of leaks, perforations, and fistulas. PCSEMS have lower migration rates as their uncovered flanges allow for anchorage into the surrounding tissue, but this consequently makes them difficult to be removed, with reported cases of esophageal perforation during the removal of the embedded PCSEMS. Stent-in-stent technique is mostly used, where a FCSEMS is placed co-axially into an existing PCSEMS causing pressure necrosis and facilitating subsequent removal of both stents. This technique, although effective, is expensive, requiring the use of a second stent. In this report, we described a series of 3 patients with embedded PCSEMS removed by a new endoscopic technique without using a second stent. In all 3 cases, the edges of stents together with lasso were completely embedded in a manner that prevented the possibility of grasping and pulling out. Stent-in-stent technique was not performed due to a temporary unavailability of FCSEMS.

By using a grasping forceps, a dissection process was initiated over the proximal flange making an opening for the scope to be inserted in the sub-stent space (the space between the esophageal wall and the stent). The process continued until a complete detachment and removal of the embedded stent was achieved.

In all 3 cases, we preferred to use a regular diagnostic scope $(9.8 \mathrm{~mm})$ to facilitate the insertion into the sub-stent space. 

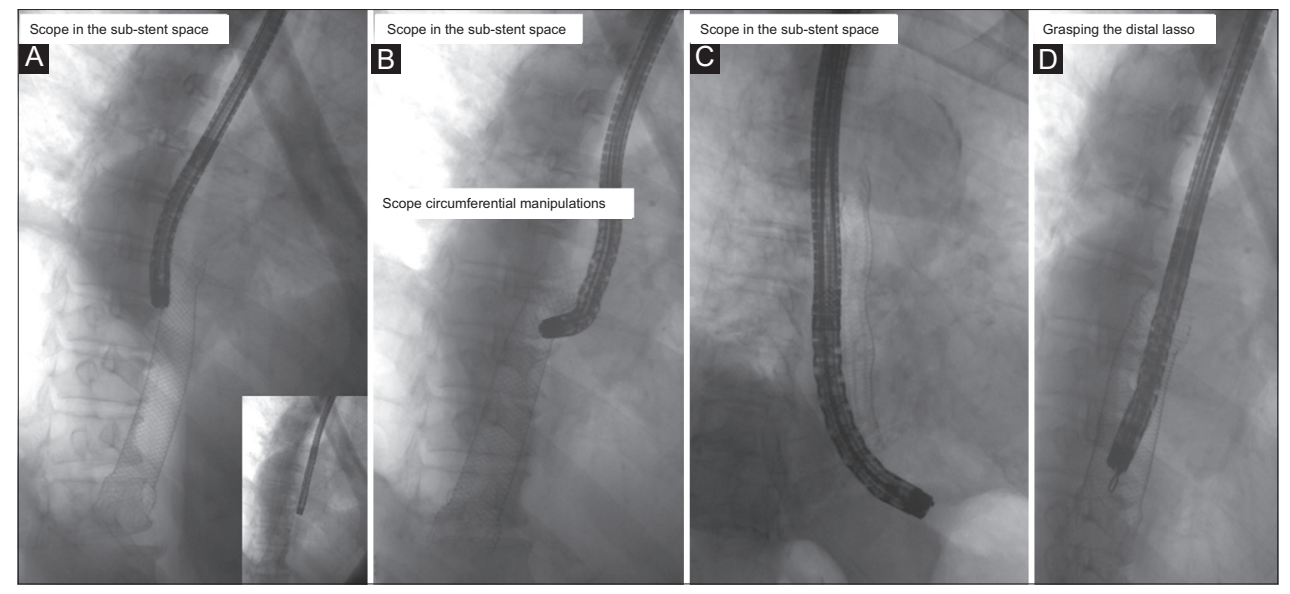

Figure 2 (A-C) Fluoroscopy of scope within the sub-stent space. (D) Grasping the distal lasso and pulling out the stent

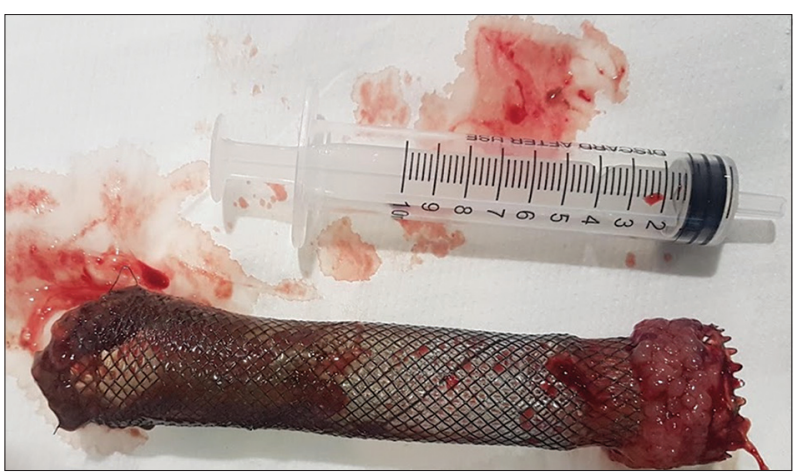

Figure 3 The removed stent (tissue ingrowth and overgrowth at both flanges)

We also found that fluoroscopy was useful in increasing the safety of the procedure by giving confidence about the correct location and position of the scope.

In conclusion, herein we described a series of 3 patients with embedded PCSEMS removed by a new endoscopic technique without using a second stent. Since this is a very small caseseries, further validation is warranted.

\section{References}

1. Kumar N, Thompson CC. Endoscopic therapy for postoperative leaks and fistulae. Gastrointest Endosc Clin N Am 2013;23:123-136.

2. Lang H, Piso P, Stukenborg C, Raab R, Jähne J. Management and results of proximal anastomotic leaks in a series of 1114 total gastrectomies for gastric carcinoma. Eur J Surg Oncol 2000;26:168-171.

3. Morales MP, Miedema BW, Scott JS, de la Torre RA. Management of postsurgical leaks in the bariatric patient. Gastrointest Endosc Clin N Am 2011;21:295-304.

4. Oh SJ, Choi WB, Song J, Hyung WJ, Choi SH, Noh SH; Yonsei Gastric Cancer Clinic. Complications requiring reoperation after gastrectomy for gastric cancer: 17-year experience in a single institute. J Gastrointest Surg 2009;13:239-245.

5. Rodrigues-Pinto E, Repici A, Donatelli G, Macedo G, et al. International multicenter expert survey on endoscopic treatment of upper gastrointestinal anastomotic leaks. Endosc Int Open 2019;7:E1671-E1682.

6. van Boeckel PG, Dua KS, Weusten BL, et al. Fully covered selfexpandable metal stents (SEMS), partially covered SEMS and selfexpandable plastic stents for the treatment of benign esophageal ruptures and anastomotic leaks. BMC Gastroenterol 2012;12:19.

7. Seven G, Irani S, Ross AS, et al. Partially versus fully covered selfexpanding metal stents for benign and malignant esophageal conditions: a single center experience. Surg Endosc 2013;27:2185-2192. 